\title{
Avaliação da mentalidade sobre o estresse em amostra do Norte e Nordeste brasileiro
}

Evandro Morais Peixoto. Universidade São Francisco

Glaucia Mitsuko Ataka da Rocha. Universidade Federal do Tocantins

José de Oliveira Franco. Universidade Cidade de São Paulo

José Maurício Haas Bueno. Universidade Federal de Pernambuco

\section{Resumo}

A mentalidade sobre o estresse é definida como as crenças que uma pessoa tem acerca das consequências positivas e negativas do estresse sobre o seu desempenho. Esta pesquisa tem como objetivo a adaptação e avaliação das propriedades psicométricas da Stress Mindset Measure (SMM). A amostra foi composta por 408 estudantes universitários (25,52 $\pm 8,72 ; 68,2 \%$ mulheres) provenientes das Regiões Norte e Nordeste do Brasil. A dimensionalidade da escala foi estimada através de Análise Fatorial Exploratória com Intercepto Randômico. Os resultados revelaram a estrutura unidimensional da SMM, corroborando a hipótese teórica, com indicadores desejáveis de precisão. Também foram estimadas correlações entre os escores da SMM e Inventário de Sintomas de Stress de Lipp, que indicaram associações negativas e de baixa magnitude entre mentalidade sobre o estresse e os sintomas de estresse, corroborando a hipótese de evidência de validade do instrumento. Os resultados sugerem adequação da versão brasileira SMM para avaliação do construto alvo.

Palavras-chave: crenças; coping; estresse; avaliação psicológica; validade.

\begin{abstract}
Assessment of stress mindset in a sample from the north and northeast regions of Brazil. Stress mindset is defined as the beliefs about the positive or negative consequences of stress on performance. This research aimed to adapt and evaluate the psychometric properties of Stress Mindset Measure (SMM). The sample consisted of 408 undergraduates $(25.52 \pm 8.72,68.2 \%$ female) from the North and Northeast Regions of Brazil. The dimensionality of the scale was estimated by Exploratory Factorial Analysis with Random Intercept. The results revealed an unidimensional structure of the SMM, corroborating the theoretical hypothesis, with good indicators of reliability. Correlations between SMM scores and Lipp's Stress Symptom Inventory were also estimated, which indicated negative and low magnitude associations between stress mindset and stress symptoms, corroborating the hypothesis of instrument validity. The results suggest the suitability of the Brazilian SMM version for the construct assessment.
\end{abstract}

Keywords: beliefs; coping; stress; psychologic assessment; validity.

\section{Resumen}

Evaluación de la mentalidad sobre el estrés en muestra del Norte y Nordeste brasileño. La mentalidad sobre el estrés se define como las creencias que una persona tiene acerca de las consecuencias positivas o negativas del estrés sobre su desempeño. Esta investigación tiene como objetivo la adaptación y evaluación de las propiedades psicométricas de la Stress Mindset Measure (SMM). La muestra fue compuesta por 408 estudiantes universitarios (25,52 $\pm 8,72,68,2 \%$ mujeres) provenientes de las Regiones Norte y Nordeste de Brasil. La dimensionalidad de la escala fue estimada a través de Análisis Factorial Exploratorio con Intercepto Randómico. Los resultados revelaron la estructura unidimensional de la SMM, corroborando la hipótesis teórica, con indicadores deseables de fiabilidad. También se estimaron correlaciones entre los escores de SMM e Inventario de Síntomas de estrés de Lipp, que indicaron asociaciones negativas y de baja magnitud entre mentalidad sobre el estrés y los síntomas de estrés, corroborando la hipótesis de evidencia de validez del instrumento. Los resultados sugieren adecuación de la versión brasileña SMM para la evaluación del constructo.

Palabras-clave: creencias; coping; estrés; evaluación psicológica; validez. 
Dentre os principais desafios enfrentados pelos estudantes de pós-graduação encontram-se aqueles relacionados aos instrumentos de medidas a serem empregados em suas pesquisas. No contexto da Psicologia, estes desafios podem estar associados à escolha dos testes ou escalas a serem utilizados para mensuração dos construtos psicológicos de interesse desses pesquisadores. Nesse momento, os pesquisadores se deparam com a demanda de instrumentos cujas propriedades psicométricas (evidências de validade, precisão e normas interpretativas) sejam conhecidas a partir de estudos realizados anteriormente, e que contemplem amostras com as mesmas características daquelas que farão parte de seus estudos, para que assim possam realizar interpretações dos escores, inferências sobre a amostra, sustentados em argumentos teóricos e empíricos (American Educational Research Association [AERA], American Psychological Association [APA], \& National Council on Measurement in Education [NCME], 2014). Esse desafio pode tomar proporções maiores num país como o Brasil devido às suas dimensões continentais, com diferenças de expressões culturais, sociais e econômicas, exigindo que estudos sobre as propriedades psicométricas dos instrumentos sejam realizados nas diferentes regiões do país e com diferentes populações (Alchieri, 2010).

Nesse sentido, importantes lacunas se caracterizam em relação à instrumentação psicológica no Brasil, haja vista que a maior parte das pesquisas são realizadas no eixo Sul-Sudeste e, consequentemente, representam as características de populações oriundas dessas regiões. Por conseguinte, regiões como Norte e Nordeste são menos representadas, tornando o desafio da escolha de instrumentos de pesquisa ainda mais evidente aos pesquisadores provenientes destas regiões, em especial quando se pretende acessar populações localizadas fora das capitais, em que o número de programas de pós-graduação é ainda mais restrito. Nessa direção, investimentos em pesquisas que permitam maior equilíbrio desta disparidade entre as regiões do Brasil são necessários. Um importante passo neste sentido foi dado pelo próprio Conselho Federal de Psicologia (CFP, 2018), por meio do Sistema de Avaliação dos Testes Psicológicos (SATEPSI), ao salientar que a atualização das normas interpretativas dos testes aprovados para uso profissional no Brasil devem "contemplar, preferencialmente, a representação demográfica de distintas regiões geopolíticas brasileiras" (Artigo 22, inciso II). A fim de oferecer contribuições para o preenchimento desta lacuna, esta pesquisa propõe a adaptação transcultural da Escala de Mentalidade sobre o Estresse (SMM) (Crum, Salovey, \& Achor, 2013) para o português brasileiro e a busca de evidências de validade e precisão do instrumento frente a uma amostra de universitários provenientes do interior das Regiões Norte e Nordeste do Brasil.

A Escala de Mentalidade sobre o Estresse adota a perspectiva de que viver em estado de estresse faz parte da vida de todo o indivíduo e que saber tirar o melhor desses estados pode ser uma saída para transformar algo que poderia ser vivenciado como exclusivamente negativo em algo positivo. Assim sendo, pode-se considerar que existem aspectos positivos do próprio estresse que podem melhorar a vida das pessoas (McGonigal, 2016). O foco exclusivo em estados negativos leva as pessoas a experimentarem sentimentos e sensações negativas, mas por outro lado, o estresse movimenta as pessoas para a vida e pode estar associado a sensações de prazer e euforia (Achor, 2012).

Assim sendo, quando se tem um stress mindset (mentalidade sobre o estresse) voltado à doença ou se alguém encara o estresse como algo que debilitará sua saúde ao longo do tempo, essa mentalidade poderá levar o indivíduo, na maior parte do tempo, a evitar situações que sejam estressantes, o que pode prejudicar seu desempenho em diversas atividades da vida. Por outro lado, quando o indivíduo tem uma mentalidade sobre o estresse a partir da qual percebe que esses estados estão melhorando seu desempenho, sua principal motivação poderá ser a de aceitar e utilizar essas situações para alcançar melhores resultados (Crum et al., 2013).

Mentalidade sobre o estresse é um construto definido como sendo as crenças que uma pessoa tem acerca das consequências que o estresse pode ter sobre o seu desempenho. Por um lado, as crenças podem ser positivas e a pessoa entende que o estresse pode melhorar seu desempenho, produtividade, saúde, bem-estar e aprendizagem, proporcionando crescimento - stres$s$-is-enhancing mindset (mentalidade positiva sobre o estresse). Por outro lado, quando as crenças sobre as consequências do estresse na vida da pessoa são negativas, a expectativa que ela tem é a de que seu desempenho será afetado negativamente pelo estresse stress-is-debilitating mindset (mentalidade negativa sobre o estresse). É importante destacar que o conceito de mentalidade sobre o estresse é uma variável distinta da severidade do estresse e da maneira como a pessoa o enfrenta. Ademais, influencia significativamente as 
respostas do indivíduo ao estresse. Ao enfrentar uma situação estressante, uma pessoa com mentalidade positiva sobre o estresse responde de maneira saudável e sofre menos influências negativas do estresse que uma pessoa com mentalidade negativa sobre o estresse (Crum, et al. 2013).

O modelo de Mentalidade sobre o estresse distingue-se do modelo Biopsicossocial (Blascovich, Seery, Mugridge, Norris, \& Weisbuch, 2004), no qual a resposta ao estresse é definida em função da avaliação cognitiva da demanda e dos recursos de enfrentamento disponíveis, a partir da qual o indivíduo define se o evento estressante é um desafio ou uma ameaça à sua integridade. A mentalidade sobre o estresse entra em ação anteriormente à avaliação cognitiva, influenciando-a.

A literatura científica tem apontado fatores ambientais e genéticos associados ao estresse. Crum, Akinola, Turnwald, Kaptchuk e Hall (2018), por exemplo, encontraram evidências de que a variação genética na catecholamina-O-methyltransferase (COMT) modifica os efeitos da mentalidade sobre o estresse em relação às respostas afetivas e cognitivas ao estresse. Assim sendo, as respostas dos indivíduos às intervenções para a mudança da mentalidade sobre o estresse podem variar de acordo com o genótipo de COMT.

Ademais, o ambiente e as ocorrências de vida também desempenham um papel importante na formação da mentalidade sobre o estresse. Um estudo realizado com adolescentes, por exemplo, ( $N=1343$; idade média=14,77; $D P=0,59$ ), mostrou que quanto maior a quantidade de eventos de vida adversos ocorridos durante um ano, maior era a percepção negativa sobre o estresse e que os adolescentes que tinham uma mentalidade positiva sobre o estresse, percebiam-no com menos intensidade (D. Park et al., 2017). Adicionalmente, há evidências de que a mentalidade positiva ou negativa em relação ao estresse traz consequências distintas para a saúde mental das pessoas. Akinola, Fridman, Mor, Morris e Crum (2016), por exemplo, encontram evidências de que o entendimento da ansiedade como algo positivo pode ser benéfico e pode minimizar os efeitos negativos do aumento de cortisol durante o desempenho de determinada atividade estressante, levando à obtenção de resultados positivos. Há, ainda, evidências de que a mentalidade negativa sobre o estresse está associada ao uso mais frequente de expressão emocional, que por sua vez, está correlacionada com altos níveis de respostas psicológicas ao estresse (Horiuchi, Tsuda, Aoki, Yoneda, \& Sawaguchi, 2018).

A psicologia dispõe de meios para intervir e modificar a mentalidade sobre o estresse, com efeitos benéficos sobre as respostas orgânicas (Crum, Akinola, Martin, \& Fath, 2017; Crum et al., 2013) assim como sobre as psicológicas (H. Park \& Hahm, 2019). Alguns autores mostraram que a exposição a vídeos informativos sobre o estresse (como potencializador ou debilitante) é capaz de alterar a mentalidade sobre o estresse (para positiva ou negativa, respectivamente) e os níveis de cortisol correspondentes, sob condição de estresse agudo (Crum et al., 2017; Crum et al., 2013). Por sua vez, H. Park e Hahn (2019) mostraram que, por meio da educação sobre cuidados com a saúde, as pessoas podem mudar a mentalidade negativa sobre o estresse para uma condição mais positiva, o que reduzia as consequências negativas causadas pelas situações estressantes, evitando a deterioração da saúde.

Apesar de o modelo biopsicossocial e a mentalidade sobre o estresse serem modelos distintos, há proposta de integração dessas perspectivas, uma vez que as intervenções fundamentadas nos dois modelos têm efeitos positivos em situações de estresse agudo. No entanto, ainda é necessário acumular mais evidências sobre cada um desses tipos de intervenção a fim de que se chegue, futuramente, a programas integrativos que facilitem o enfrentamento ativo do estresse (Jamieson, Crum, Goyer, Marotta, \& Akinola, 2018).

Com a finalidade de se avaliar a mentalidade sobre o estresse e verificar a eficácia das intervenções para promover a mudança de mentalidade, foi elaborada uma medida, a Stress Mindset Measure (SMM) (Crum et al., 2013). O processo de elaboração da SMM teve início com a geração de itens por um grupo focal formado por alunos de graduação, pós-graduação e pós-doutorandos do Health, Emotional, and Behavioral Laboratory, da Universidade de Yale. Duas versões da escala foram criadas: uma delas é referente às crenças sobre a natureza do estresse de maneira geral (SMM-G) e a outra, relativa às crenças sobre a natureza do estresse no contexto de estressores específicos (SMM-S). As duas escalas foram aplicadas em 388 funcionários de uma grande instituição financeira internacional nos Estados Unidos da América (EUA). A amostra foi escolhida em razão de a companhia passar por importante processo de redução de atividades e reestruturação em resposta à recessão econômica. Os instrumentos aplicados no estudo foram: 
Social Readjustment Rating Scale (SRRS), que avalia a quantidade de estresse vivenciada pela pessoa; Brief $C O P E$, que avalia as estratégias de enfrentamento do estresse; Perceived Stress Scale (PSS), que mensura a percepção do estresse vivenciado pela própria pessoa; Life Orientation Test (LOT-R), que avalia a disposição ao otimismo; e Freiburg Mindfulness Inventory (FMI), que avalia a capacidade de ter consciência do presente combinada ao não julgamento dessas percepções. Os resultados desse estudo indicaram que ambas as escalas têm bom níveis de consistência interna alta (a Crombach $=0,80$ para a SMM-S e 0,86 para a SMM-G). Além disso, as respostas a ambas apresentaram uma distribuição normal. Idade e sexo não se relacionaram aos escores da SMM, sendo que SMM-S e SMM-G estavam correlacionadas entre si $(r=0,61$, $p<0,001)$. As correlações encontradas entre a SMM e as demais medidas foram significativas, na direção esperada e variaram entre $(r=0,77$ e $r=0,86, p<0,05$ e $p<0,01$ ) (Crum et al., 2013).

A Análise Fatorial Confirmatória indicou a unifatorialidade da SMM, para a qual as cargas dos itens variaram entre 0,56 e 0,78 . A fim de confirmar se a mentalidade sobre o estresse seria um construto independente das demais variáveis avaliadas, procederam à Análise de Equações Estruturais, comparando modelos em que a SMM seria um constructo independente e outros modelos em que a SMM avaliaria os mesmos construtos das outras medidas. As análises indicaram que o modelo no qual a SMM é considerada um construto independente foi o melhor [CMIN (14) = 38,07, $p<0,01$ ); $\operatorname{CFI}(0,97)$; RMSEA $(0,06)$ e AIC $(98,07)]$ quando comparado aos demais. Foi também realizado um estudo de validade utilizando como critérios externos medidas de Saúde (Mood and Anxiety Symptom Questionnaire MASQ e Health Days Measures - HD - do Center for Disease Control and Prevention), Desempenho (Work Performance Scale - WPS) e Qualidade de Vida (Quality of Life Inventory - QOLI). Os coeficientes de correlação variaram entre $(r=0,77$ e $r=0,87, p<0,05$ e $p<0,01)$ (Crum et al., 2013).

Com base no exposto, entende-se que o estresse pode ter um impacto bastante significativo na vida das pessoas, e a forma como esse fator é percebido, pode potencializar ou amenizar esse impacto. No entanto, há poucos instrumentos com boas propriedades psicométricas para a medida de estresse que permitam verificar a eficácia de intervenções que visem à modificação da mentalidade sobre o estresse. E, até onde se sabe, não há instrumentos para a mensuração da forma como o estresse é percebido no contexto cultural brasileiro. Por isso, o objetivo desta pesquisa foi adaptar a SMM (Crum et al., 2013), para português brasileiro e estimar as primeiras evidências de validade com base na estrutura interna, relação com variável externa e precisão.

\section{Método}

\section{Participantes}

A amostra, por conveniência, foi composta por 408 estudantes universitários com idades que variavam entre 17 e 61 anos $(M=25,52 \pm 8,72)$, dos quais $68,2 \%$ eram mulheres. Em relação ao estado civil $72,9 \%$ se autodeclararam solteiros, $16,8 \%$ casados, $5,9 \%$ divorciados e 4,4\% não forneceram informações. Quanto às instituições de origem, os participantes eram provenientes de universidades públicas localizadas no interior de Pernambuco e interior de Tocantins, e representantes de diferentes cursos de graduação: $58,4 \%$ psicologia, 15,8\% medicina, 3,4\% matemática, e curso de pós-graduação $22,4 \%$.

\section{Instrumentos}

Stress Mindset Measure-SMM - (Crumetal., 2013). É um instrumento de autorrelato composto por oito itens, quatro positivos (ex. Os efeitos do estresse são positivos e podem ser úteis) e quatro negativos (ex. Vivenciar o estresse esgota minha saúde e vitalidade). Os itens da SMM são respondidos por meio de escala do tipo likert, em que as respostas variam de $0=$ Discordo fortemente até 4= Concordo fortemente. Estudos de avaliação das propriedades psicométricas da escala demostraram a adequação de estrutura unifatorial, bem como níveis desejáveis de precisão, com coeficientes alfa de Crombach que variaram entre 0,80 e 0,86. Para composição do escore final em mentalidade sobre o estresse os itens negativos devem ser invertidos e todos os itens somados, valores mais elevados desses escores indicam maiores níveis de mentalidade positiva.

Inventário de Sintomas de Stress para Adultos ISSL - (Lipp, 2000). O ISSL avalia os sintomas de estresse e possibilita a classificação da fase do estresse em que a pessoa se encontra: a) Alerta: uma fase positiva do estresse, com aumento da produção de adrenalina, levando a pessoa ao estado de alerta para lidar com situações específicas. Apesar da presença de taquicardia, tensão, insônia, a pessoa sente-se capaz de lidar com as situações; b) Resistência: baixa na produtividade, 
aumento da produção de cortisol, vulnerabilidade imunológica, utiliza da energia destinada a outras funções do organismo, para tentar restabelecer a homeostase do organismo; c) Quase exaustão: aumento excessivo da produção de cortisol, tendo efeitos negativos na defesa imunológica, com o surgimento de doenças, ansiedade, variabilidade emocional; d) Exaustão: considerada a fase patológica e negativa do estresse, pois provoca grande desequilíbrio interior, aparecimento de quadros depressivos, falta de concentração, aumento da impulsividade. Doenças graves podem começar a surgir nessa fase. O ISSL possibilita identificar a fase de estresse em que a pessoa se encontra, bem como se são predominantes os sintomas físicos ou psicológicos.

\section{Procedimentos}

Tradução e adaptação da SMM para o Português brasileiro. Inicialmente entrou-se em contato com um dos autores da SMM, Dra. Alia Crum, do Mind and Body Lab, da Universidade de Stanford, que disponibilizou a versão original em inglês. $O$ instrumento foi traduzido para o português brasileiro por três tradutores bilíngues português-inglês, sendo um deles tradutor profissional e dois deles pesquisadores da área de Psicologia com conhecimento sobre estresse e avaliação psicológica. Não foram encontradas dificuldades para a construção de uma síntese das traduções, uma vez que os itens são claros e objetivos e a escala é breve. Em etapa subsequente, de back translation, a versão síntese em português foi traduzida para a língua inglesa, por um tradutor profissional, e enviada para a equipe do Mind and Body Lab da Universidade de Stanford, a fim de que se confirmasse a adequação dos itens da SMM, uma vez retraduzida. Feitos os últimos acertos na redação dos itens, a SMM foi aplicada a um grupo de cinco profissionais da área de Tecnologia da Informação, no município de São Paulo - SP, a fim de se verificar sua compreensão. Além de responderem a SMM, os participantes foram questionados acerca da sua compreensão dos itens.

\section{Considerações Éticas}

A aplicação da SMM e o ISSL ocorreram após a aprovação do projeto pelo Comitê de Ética da Universidade Ibirapuera UNIB sob número de protocolo (CAAE 73179217.2.00005597). Para coleta de dados foram realizados contatos com os diretores dos campi universitários, os quais consentiram formalmente e, portanto, possibilitaram o contato com os possíveis participantes. Os instrumentos foram administrados de forma coletiva, em salas de aula, após o assentimento formal por parte dos professores, explicação dos objetivos da pesquisa aos estudantes e assinatura do Termo de Consentimento Livre e Esclarecido, por parte dos estudantes que concordavam em participar da pesquisa.

\section{Análise de Dados}

Para a estimação do número de fatores a serem extraídos para versão brasileira da SMM, empregou-se diferentes métodos de retenção de fatores: Critério Gutmman-Kiser (eigenvalue superior a um), Análise Paralela e método Hull (Lorenzo-Seva, Timmerman, \& Kiers, 2011). Posteriormente, a solução fatorial foi estimada por meio da Análise Fatorial Exploratória (AFE), tendo como base matrizes de correlação policórica e método de estimação apropriado ao nível de mensuração ordinal, Unweighted Least Squares (ULS). Essas análises foram realizadas com apoio do software estatístico Factor v. 10.8.04 (Lorenzo-Seva \& Ferrando, 2013).

Além da AFE recorreu-se a um modelo de Análise Fatorial com Intercepto Randômico (AFE-IR), conforme proposto por Maydeu-Olivares e Steenkamp (2018) com o objetivo de verificar a influência de vieses de resposta na estimação da estrutura interna da SMM. A AFE-IR pode ser considerada uma extensão da modelo fatorial comum. Conforme extensamente descrito na literatura, no modelo fatorial um conjunto de variáveis observadas (itens de um teste) são expressas como uma função linear de um conjunto menor de fatores comuns (variáveis não observadas) e o erro associado à medida. Além disso, assume-se que a relação entre cada variável observada (por exemplo, pontuação do item) e as variáveis não observadas são lineares e que, portanto, os coeficientes lineares (interceptos e cargas fatoriais) são invariantes, ou seja, não diferem entre os respondentes do teste. Considerando, um conjunto de $\mathrm{N}$ indivíduos a um conjunto de $p$ itens, o modelo fatorial $y_{i j}$ pode ser descrito com:

Onde:

$$
y_{i j}=\mu i_{+} \lambda_{i} \eta_{j+} e_{i j}
$$

$\mu_{i}=$ intercepto do item $\mathrm{i}$

$\lambda_{i}=$ carga fatorial do item $\mathrm{i}$

$\eta_{i j}=$ vetor do fator comum dos participantes $j$ 's

$\mathrm{e}_{i j}=$ termo de erro para o participante $j$ no item $i$

Ressalta-se que, no modelo apresentado na equação supracitada, o fator comum " $\eta$ " e o termo de erro "e", são acompanhados dos subscritos "j" e, portanto, referem-se a variáveis randômicas que mudam entre os participantes. Estas variáveis contrastam com 
as variáveis intercepto " $\mu_{i}$ " e carga fatorial " $\lambda_{i}$ ", que não incluem o subscrito "j" e, portanto, são variáveis fixas, invariantes entre os participantes (Maydeu-Olivares \& Coffman, 2006). Na proposta do intercepto randômico a suposição de um intercepto comum a todos os respondentes é relaxada, permitindo que o intercepto varie de respondente para respondente. Para tanto, propõe-se a modelagem de um fator geral não correlacionado aos fatores que representam os construtos-alvo. Como todas as cargas deste fator adicional são fixadas em 1, o escore estimado pode ser interpretado como uma espécie de "escore de viés de resposta", refletindo o quanto os respondentes estão propensos a endossar o item, independentemente do conteúdo representado por ele (Maydeu-Olivares \& Steenkamp, 2018; Valentini, 2017).

Desta forma, a modelagem do intercepto randômico possibilita o conhecimento dos itens mais influenciados pelo viés de resposta, bem como minimizar os efeitos destes vieses na compreensão da estrutura interna dos instrumentos de medida, elevando a precisão dos instrumentos na estimação das variáveis latentes. Vale ressaltar que a omissão deste controle pode levar a estimativas espúrias da estrutura interna dos instrumentos e, consequentemente, das estimativas características das pessoas. Desta forma, os modelos estimados foram avaliados a partir dos índices de adequação recomendados por Muthén e Muthén (2012): $\chi^{2}, d f, \chi^{2} / d f$, RMSEA, CFI e TLI. Estabeleceu-se como parâmetro os seguintes valores de referência: $\chi^{2} / d f<5$, RMSEA $<0,08$, CFI e TLI $>0,90$. Esse procedimento foi realizado com base no software estatístico Mplus 7,2 (Muthén \& Muthén, 2012)
Ainda em relação à estrutura interna, realizou-se a avaliação dos indicadores de precisão da versão brasileira da SMM. Para tanto, recorreu-se à estimação dos coeficientes alfa de Cronbach e ômega de McDonald. Conforme indicado pela literatura especializada, tomou-se, como referência, índices iguais ou superiores a 0,70 (Marôco, 2010).

A última etapa da presente pesquisa consistiu na avaliação de evidências de validade com base na relação com variáveis externas (percepção do estresse), sob a hipótese de que mentalidade sobre o estresse e percepção dos sintomas do estresse são construtos distintos, contudo negativamente relacionados, uma vez que pessoas com mentalidade positiva sobre o estresse tendem a sofrer menos os impactos negativos do estresse (Crum et al., 2013). Para tanto, estimou-se coeficientes de correlação de Pearson entre a SMM e ISSL, tomando como referências níveis de significância inferiores a 0,05 (Tabachnick \& Fidell, 2012). Esta análise foi realizada com apoio do software estatístico JASP v 0.9 (JASP Team, 2018).

\section{Resultados}

Antes de submeter os dados à $\mathrm{AFE}$, verificou-se os indicadores de adequação dos itens que compõem a SMM para a realização deste procedimento estatístico. Os resultados, índice $\mathrm{KMO}=0,8472$ e $\chi^{2}$ de Bartlett $=(28)$ $1019,7, p<0,001$, indicaram adequação da matriz de correlação entre os itens para a realização de análises fatoriais. Desta forma, realizou-se a avaliação dos métodos de retenção de fatores e os resultados são apresentados na Tabela 1, em que se verificam os eigenvalues, resultados referentes à AP e método Hull.

Tabela 1. Métodos de Retenção de Fatores

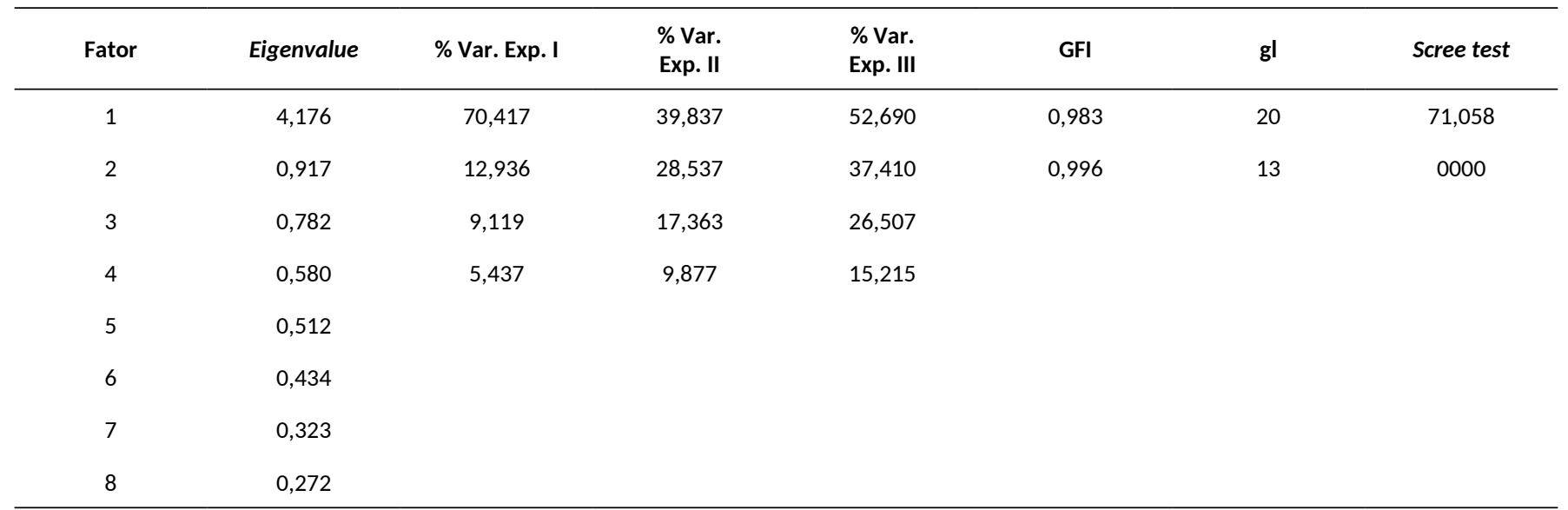

Nota: \% Var. Exp. I = Porcentagem de variância explicada provenientes dos dados reais; \% Var. Exp. II = porcentagem de variância média provenientes dos dados aleatórios; $\%$ Var. Exp. III= porcentagem de variância explicada alocada no percentil 95 dos dados aleatórios. 
Conforme observado na Tabela 1, todos os métodos sugerem a adequação de estrutura unifatorial, haja vista que apenas o primeiro fator apresentou eigenvalue superior a 1 , valor de variância explicada superior a variância explicada média, e superior ao valor de variância explicada alocado no percentil 95, provenientes das matrizes estimadas simuladas aleatoriamente, e o melhor equilíbrio entre índices de ajuste e graus de liberdade (qualidade dos índices de ajuste $=(20) 0,983$, e índices Scree teste $=71,058$, quando avaliado o método Hull.

Além disso, a solução unifatorial explicou 52,02\% da variância total dos dados. O modelo fatorial da SMM é apresentado na Tabela 2, na qual são apresentadas as cargas fatoriais padronizadas, estimadas por meio da AFE e AFE-IR, e os indicadores de consistência interna.

Tabela 2. Estrutura Fatorial da SMM Estimada por Meio de AFE E AFE-IR

\begin{tabular}{|c|c|c|c|c|c|}
\hline \multirow[b]{2}{*}{ Itens } & \multirow{2}{*}{$\begin{array}{c}\text { AFE } \\
\text { F1 }\end{array}$} & \multicolumn{2}{|c|}{ AFE-IR } & \multicolumn{2}{|c|}{ AFE-IR / 5} \\
\hline & & F1 & IR & F1 & IR \\
\hline 1 & $-0,588$ & $-0,571$ & $-0,100$ & $-0,593$ & 0,112 \\
\hline 2 & 0,639 & 0,645 & 0,108 & 0,620 & 0,198 \\
\hline 3 & $-0,627$ & $-0,611$ & 0,021 & $-0,656$ & 0,384 \\
\hline 4 & 0,756 & 0,750 & 0,274 & 0,804 & 0,264 \\
\hline 5 & $-0,700$ & $-0,785$ & 0,697 & & \\
\hline 6 & 0,683 & 0,677 & 0,258 & 0,708 & $-0,078$ \\
\hline 7 & $-0,770$ & $-0,748$ & 0,089 & $-0,707$ & 0,014 \\
\hline 8 & 0,746 & 0,737 & 0,163 & 0,748 & 0,046 \\
\hline$a$ & & 0,868 & & & \\
\hline$\omega$ & & 0,869 & & & \\
\hline
\end{tabular}

Nota: AFE = Análise Fatorial Exploratória; AFE-IR Análise Fatorial

Exploratória com Intercepto Randômico; IR = Fator intercepto randômico.

Através dos resultados apresentados na primeira coluna da Tabela 2, observa-se que os itens apresentaram cargas fatoriais em acordo com a perspectiva teórica descrita pelo modelo de mentalidade sobre estresse. Desta forma, os itens 1, 3, 5 e 7 apresentaram cargas fatoriais negativas corroborando as hipóteses iniciais, uma vez que foram formulados teoricamente para acessar a percepção dos respondentes sobre os efeitos negativos do estresse em diferentes aspectos da vida: saúde e vitalidade, crescimento pessoal, e desempenho e produtividade. Além disso, observa-se que todos os itens apresentaram cargas fatoriais altas, entre $-0,588$ (item 1 ) e -0,770 (item 7), demonstrando-se como bons representantes do construto avaliado pelo fator.

Os índices alfa de Cronbach e ômega de McDonald sugerem bom nível de consistência interna da estrutura observada, dando suporte assim, à precisão da versão brasileira da SMM. Os resultados observados até esta etapa dão suporte às primeiras evidências de validade com base na estrutura interna e precisão da versão brasileira do instrumento alvo da presente pesquisa (SMM). Contudo, avaliou-se também os indicadores de ajustamento do modelo de medida (estrutura interna) fornecidos pelo software Mplus. Mesmo em se tratando da análise de modelos exploratórios, podem ser classificados como inadequados: $\chi^{2}=175,967$; $d f=20 ; \chi^{2} / d f=8,80, p<0,001 ; C F I=0,937 ; \mathrm{TLI}=0,911$; RMSEA $=0,138$ (I.C. $90 \%=0,120-0,157$ ).

Em decorrência disso, os mesmos dados foram avaliados por meio da AFE-IR para avaliação de itens mais influenciados por vieses de resposta (aquiescência). Observa-se na segunda (F1) e terceira (IR) colunas da Tabela 2 o modelo fatorial estimado pela AFE-IR. Neste caso, a coluna F1 representa a carga fatorial padronizada do item no fator mentalidade sobre o estresse, enquanto a coluna IR representa a carga fatorial em "viés de resposta/aquiescência". Pode-se observar que após a estimação do intercepto randômico, mudanças importantes foram observadas em relação ao item 5 (Vivenciar o estresse restringe meu aprendizado e meu crescimento), que apresentou melhora na carga fatorial padronizada (subindo de $-0,700$ para $-0,785)$, acompanhada, no entanto, de uma carga também elevada no "viés de resposta/aquiescência", igual a 0,697. Tais resultados sugerem a forte influência da aquiescência na resposta a esse item, ou 
seja, refletem o quanto os respondentes endossam este item, independentemente do seu conteúdo. A título de comparação, são apresentados os índices de ajuste do modelo, que apresentam uma melhora substancial $\left(\chi^{2}=80,429 ; d f=13 ; \chi^{2} / d f=6,18, p<0,001 ; C F I=0,970\right.$; $\mathrm{TLI}=0,936$; RMSEA $=0,117$ (I.C. $90 \%=0,093-0,142)$ ), mas ainda superiores aos esperados para um bom ajuste nos índices $\chi^{2} / d f$ e RMSEA.

Por fim, optou-se pela realização de uma nova AFE-IR com a exclusão do item 5 do modelo e foram observados índices de ajustamento classificáveis como muito bons: $\left(\chi^{2}=25,793 ; d f=8 ; \chi^{2} / d f=3,22, p<0,001\right.$;
$\mathrm{CFI}=0,991 ; \mathrm{TLI}=0,977 ; \mathrm{RMSEA}=0,064$ (I.C. $90 \%=0,033-$ 0,081 ), bem como itens com bons níveis de carga fatorial no fator alvo (stress mindset), entre -0,593 (item 1) e 0,804 (item 4), e valores aceitáveis de carga no fator intercepto randômico. Mesmo com a exclusão de um item do modelo original a estrutura manteve bom nível de consistência interna, sugerindo bons indicadores de precisão para o instrumento, ambos com valores iguais 0,85 .

A última etapa da pesquisa buscou avaliar a relação entre a SMM e Inventário de Sintomas de Stress de LIPP (ISSL), cujos índices de correlação de Pearson entre as escalas são apresentados na Tabela 3.

Tabela 3. Índices de Correlação Pearson entre a SMM e o ISSL (N=200)

\begin{tabular}{|c|c|c|c|c|c|c|}
\hline & SMM & Q1F & Q1P & Q2F & Q2P & Q3F \\
\hline Q1F &,- 076 & & & & & \\
\hline Q1P &, $226 * *$ &,- 031 & & & & \\
\hline Q2F &,$- 207^{* *}$ & , $665^{* *}$ &,- 097 & & & \\
\hline Q2P &,$- 274^{* *}$ & ,494** &,- 008 & ,620"* & & \\
\hline Q3F &,- 066 &, $572^{* *}$ &,- 030 &, $586^{* *}$ & ,482* & \\
\hline Q3P &,$- 236 * *$ &, $506^{* *}$ &,- 032 & ,678** &, $751^{* *}$ &, $581^{* *}$ \\
\hline
\end{tabular}

Nota: Q1= Alerta; Q2= Resistência; Q3= Exaustão; F= Físico; P= Psicológico;

$\cdot=p<0,001$.

Conforme observado na Tabela 3, primeira coluna, os índices de correlação entre a SMM e o ISSL foram significativos e de magnitudes fracas. Quanto à direção destes índices de correlação, observou-se correlação positiva entre mentalidade sobre estresse e sintomas psicológicos referentes à fase de alerta, bem como associação negativa, entre mentalidade sobre estresse e sintomas físicos e psicológicos referentes às fases de resistência/quase exaustão e sintomas psicológicos referentes à fase de exaustão.

\section{Discussão}

Nesta pesquisa teve-se como objetivo adaptar a SMM (Crum et al., 2013), para o português brasileiro e estimar as primeiras evidências com base na estrutura interna, relação com variável externa e precisão (AERA et al., 2014), instrumento que se baseia no modelo unifatorial da mentalidade sobre o estresse, que corresponde às crenças negativas e positivas que uma pessoa desenvolve a respeito das consequências do estresse sobre o seu desempenho. De forma geral, os resultados indicaram a pertinência da versão traduzida e adaptada da SMM para mensuração da mentalidade sobre o estresse em estudantes brasileiros, o que disponibiliza aos pesquisadores uma medida para avaliar intervenções cujo objetivo principal é o de promover a mudança na mentalidade sobre o estresse (Crum et al., 2013) e, por conseguinte, potencializar os resultados benéficos em detrimento dos negativos, principalmente em situações nas quais as mudanças externas são muito difíceis de serem realizadas.

A partir dos métodos de retenção de fator e da análise fatorial convencional verificou-se a reprodutibilidade do modelo unifatorial e atestou-se a adequação do conjunto de itens que compõem a SMM, conforme originalmente proposto por Crum et al. (2013), haja vista que todos os itens apresentaram boas cargas fatoriais, iguais ou superiores a 0,588, bem como níveis desejáveis de precisão (Marôco, 2010; Tabachnick \& Fidell, 2012), equivalentes aos observados no estudo da versão original. Vale ressaltar que o emprego do construto mentalidade sobre o estresse na literatura cientifica em psicologia é recente (Kilby \& Sherman, 2016) e, embora versões do instrumento tenham sido adaptadas para outras nacionalidades como Chinesa, Húngara, Italiana e Polonesa (ver https://mbl.stanford. edu/materials-measures/translations), ainda não se conta com estudos de avaliações das propriedades 
psicométricas dessas versões, o que impossibilita a comparação das propriedades observadas na presente pesquisa com outros estudos.

Tais resultados assegurariam as primeiras evidências de validade e precisão da SMM para o contexto brasileiro (AERA et al., 2014); contudo a reavaliação dos modelos de medida através da AFE-RI (Maydeu-Olivares \& Coffman, 2006; Valentini, 2017) indicou a forte influência de viés de resposta ao item " 5 -Vivenciar o estresse restringe meu aprendizado e meu crescimento", sugerindo uma tendência sistemática de os respondentes endossarem o item, ou seja, escolherem pontuações altas entre as opções de respostas na escala likert, independente do conteúdo do item (Paulhus, 1991). Esse fenômeno tem sido explicado na literatura psicométrica de diferentes pontos de vista (Wetzel, Böhnke, \& Brown, 2016); dentre eles destacam-se os conceitos de desejabilidade social e aquiescência.

De acordo com Wetzel et al. (2016) a desejabilildade social corresponde à intenção dos respondentes em oferecer respostas socialmente desejáveis, enquanto a aquiescência corresponde a uma tendência em concordar com o conteúdo dos itens. Diferentes questões metodológicas podem influenciar esses fenômenos, como a elaboração de item com alta carga valorativa ou mesmo itens elaborados com conteúdo de difícil entendimento. Compreende-se que o item não apresenta alta carga valorativa, haja vista que conteúdo semelhante é apresentado no item "2.-Vivenciar o estresse facilita meu aprendizado e meu crescimento", e mostrou-se pouco influenciado por viés de resposta, haja vista que sua carga fatorial no fator mentalidade sobre o estresse foi de 0,645 , enquanto no intercepto randômico foi de 0,108. Desta forma, trabalha-se com a possibilidade de que a redação do item negativo, com a utilização de uma palavra pouco convencional "restringe", pode ter dificultado o entendimento do item levando os respondentes a endossá-lo sem considerar de fato o seu conteúdo.

Com base nestes resultados, sugere-se a substituição da palavra "restringe" por "prejudica", guardando assim o sentido atribuído ao item originalmente e oferecendo aos respondentes uma palavra mais familiar e, portanto, de fácil compreensão. Desta forma, sugere-se que novos estudos sobre evidências de validade da SMM sejam realizados no contexto brasileiro considerando a seguinte redação para o item 5: "-Vivenciar o estresse prejudica meu aprendizado e meu crescimento". Adicionalmente, destaca-se a potencialidade dos procedimentos estatísticos que buscam modelar a influência de vieses de resposta na estimação de estrutura interna de medidas psicológicas (Maydeu-Olivares \& Coffman, 2006; Valentini, 2017; Zanon, Lessa, \& Zanon, 2018). Por meio da AFE-IR foi possível obter cargas fatoriais dos itens mais adequadas, haja vista que com a modelagem do viés de resposta o item 5 apresentou melhora significativa na carga fatorial no fator alvo da escala, o modelo apresentou melhores indicadores de ajustamento, e foi possível discutir, a partir desses resultados, a melhora na redação do item. Ressalta-se que tais características das propriedades psicométricas da SMM não foram passíveis de compreensão a partir da AFE convencional, que indicou adequação de todos os itens.

A AFE-IR conforme proposta por Maydeu-Olivares e Coffman (2006), tem sido empregada no contexto brasileiro para avaliação da influência do viés de resposta na estrutura interna de instrumentos multidimensionais. Pesquisas recentes sugerem a adequação deste método para estimação de estruturas mais coerentes com a perspectiva teórica na avaliação da personalidade (Big Five Inventory) (Zanon et al., 2018) e dados simulados com organização multifatorial (Valentini, 2017). Nessa direção, a presente pesquisa demostra a adequação deste método para avaliação de escalas unifatoriais, e a potencialidade das informações obtidas a partir da AFE-IR para o refinamento destes instrumentos de medidas psicológicas. Desta forma, sugere-se que o primeiro objetivo da presente pesquisa foi satisfatoriamente alcançado: a obtenção das primeiras evidências de validade com base na estrutura interna da versão brasileira da SMM (AERA et al., 2014).

Em relação aos indicadores de precisão da SMM, mesmo com a exclusão do item 5 , os índices alfa de Cronbach e ômega de McDonald (0,852 e 0,854, respectivamente) indicaram valores coerentes com aqueles observados no estudo original, coeficientes alfa de Cronbach que variaram entre 0,80 e 0,86 (Crum et al., 2013). Desta forma, sugere-se que o segundo objetivo da presente pesquisa também foi satisfatoriamente alcançado, podendo-se afirmar que a versão brasileira da SMM apresentou índices desejáveis de precisão (AERA et al., 2014).

A última etapa da presente pesquisa constituiu na estimação de evidência de validade com base na relação com variável externa, para tanto foram estimadas correlações entre o escore fatorial estimado a partir da AFE-IR para SMM e os escores do ISSL. Neste sentido, os resultados encontrados corroboram os achados 
anteriores de Crum et al. (2013) e Kilby e Sherman (2016), nos quais também foram encontradas associações negativas de baixa magnitude entre mentalidade sobre o estresse e sintomas de estresse, o que significa que os indivíduos que se percebem menos estressados ou que percebem menos a presença de sintomas de estresse são mais frequentemente aqueles que têm uma mentalidade positiva sobre o estresse. Ademais, os resultados trazem evidências adicionais de que a mentalidade sobre o estresse seja um construto distinto da percepção de estresse e de seus sintomas, o que confere as primeiras evidências de validade discriminante para a versão brasileira da SMM.

Outro resultado que fortalece estas evidências é a associação positiva observada entre a mentalidade sobre estresse e os sintomas psicológicos da fase de alerta. Ressalta-se que esta é a primeira fase do estresse, vista como uma etapa positiva em que as pessoas se sentem com energia e vigor para realizações (Lipp, 2000). Tais resultados corroboram as expectativas teóricas de que uma pessoa que tenha uma mentalidade positiva de estresse tende a aceitar desafios e a utilizar o estresse a fim de obter resultados que a levem ao crescimento e a um melhor desempenho (Crum et al., 2013).

Estudos têm indicado os diferentes impactos da mentalidade sobre o estresse na vida das pessoas (Akinola et al., 2016; Crum et al., 2013; Horiuchi et al., 2018). A proposta teórica da mentalidade sobre o estresse sugere que as crenças positivas sobre o estresse levam a resultados positivos diante do envolvimento com eventos estressores. Da mesma forma, o modelo prevê que a percepção de um evento estressor como um desafio leva a pessoa a adotar estratégias de enfrentamento e a obter resultados positivos, enquanto as percepções negativas do evento estressor como ameaçador invocam estratégias e resultados negativos (Crum et al., 2013; Kilby \& Sherman, 2016).

É importante observar que a mentalidade sobre o estresse mostrou ser um construto possível de ser modificado por meio de intervenção psicoeducativa, disponibilizada via internet (Crum et al., 2013; H. Park \& Hahn, 2019). São intervenções simples, realizadas à distância e cujos resultados podem ser avaliados pela SMM. Contar com a versão brasileira da SMM, com bons indicadores de validade e precisão e, portanto, com boas capacidades de mensuração desse construto psicológico, pode ser visto como um passo importante ao se disponibilizar ao contexto brasileiro, em especial para as Regiões Norte-Nordeste, uma ferramenta adequada a compor o instrumental de pesquisadores que pretendem acompanhar o desenvolvimento dessas características em futuras pesquisas.

\section{Considerações finais}

Esta pesquisa apresentou a adaptação para o português brasileiro e avaliação das primeiras evidências de validade com base na estrutura interna, relação com outras variáveis e precisão da SMM. O instrumento se caracteriza como uma medida breve, de fácil e rápida aplicação para avaliação da mentalidade sobre o estresse em amostra de estudantes brasileiros das Regiões NorteNordeste. Vale ressaltar que grande parte dos estudos voltados para adaptação de instrumentos de medida ao contexto brasileiro concentram amostras oriundas do eixo Sul-Sudeste. Nessa direção, faz-se necessário investimento para que amostras oriundas de outras regiões do país sejam acessadas, pois as lacunas tornam-se mais evidentes quando pensadas as populações alocadas no interior dos estados localizados nas Regiões Norte e Nordeste. Espera-se que este trabalho tenha trazido algumas contribuições para o preenchimento destas lacunas ou, ao menos, possam ecoar em reflexões sobre a aplicabilidade dos instrumentos de avaliação psicológica fora do eixo Sul-Sudeste e motivar novas pesquisas em outras regiões geográficas além dessas.

Destaca-se algumas das limitações da presente pesquisa, como o fato de contar com uma amostra não probabilística. Por conseguinte, recomenda-se cautela na generalização dos resultados encontrados. Outro aspecto a ser salientado refere-se à restrição da coleta de dados apenas por meio do procedimento lápis e papel, fato que limita o número de respondentes. Entende-se que a utilização de procedimentos eletrônicos pode contribuir para a superação destas limitações, sendo tal ferramenta indicada para possibilitar maior representatividade das amostras em países de extensão continental, como o Brasil. Por fim, sugere-se a realização de novos estudos em função da modificação proposta no item 5 , a fim de verificar se os resultados encontrados serão mantidos. Além disso, sugere-se estudos que não se restrinjam a amostras compostas por universitários, mas que abarquem pessoas em outras atividades produtivas ou situações, possibilitando a ampliação da aplicação do instrumento a outros contextos. 
Avaliação da mentalidade sobre o estresse em amostra do norte e nordeste brasileiro

\section{Referências}

Achor, S. (2012). O jeito Harvard de ser feliz. São Paulo: Saraiva.

Akinola, M., Fridman, I., Mor, S., Morris, M. W., \& Crum, A. J. (2016). Adaptive appraisals of anxiety moderate the association between cortisol reactivity and performance in salary negotiations. PLOSONE, 11(12): e0167977. doi: 10.1371/journal.pone.0167977

Alchieri, J. (2010). Análise dos dados demográficos das normas brasileiras de instrumentos psicológicos empregados na avaliação da personalidade. Revista Psicologia e Saúde, 2(1) (2010), 56-63 56. doi: $10.20435 /$ pssa.v2i1.36

American Educational Research Association - AERA, American Psychological Association - APA, \& National Council on Measurement in Education - NCME (2014). Standards for educational and psychological testing. Washington, DC: AERA.

Blascovich, M., Seery, D., Mugridge, C. A., Norris, R. K., \& Weisbuch, M. (2004). Predicting athletic performance from cardiovascular indexes of challenge and threat. Journal of Experimental Social Psychology, 40, 683-688. doi: 10.1016/j.jesp.2003.10.007

Conselho Federal de Psicologia - CFP. (2019). Resolução 09/2018. Recuperado de

http://satepsi.cfp.org.br/docs/Resolu\%C3\%A7\%C3\%A3o-CFP-n\%C2\%BA09-2018-com-anexo.pdf

Crum, A. J., Akinola, M., Martin, A., \& Fath, S. (2017). The role of stress mindset in shaping cognitive, emotional, and physiological responses to challenging and threatening stress. Anxiety, Stress \& Coping, 30(4), 379-395. doi: 10.1080/10615806.2016.1275585

Crum A. J., Akinola M., Turnwald, B. P., Kaptchuk, T. J., \& Hall, K. T. (2018). Catechol-O-Methyltransferase moderates effect of estresse mindset on affect and cognition. PLOS ONE 13(4): e0195883. doi: 10.1371/ journal. pone.0195883

Crum, A. J., Salovey, P., \& Achor, S. (2013). Rethinking estresse:The role of mindsets in determining the estresse response. Journal of Personality and Social Psychology, 104(4), 716-33. doi: 10.1037/a0031201

Horiuchi, S., Tsuda, A., Aoki, S., Yoneda, K., \& Sawaguchi, Y. (2018). Coping as a mediator of the relationship between estresse midset and psychological estresse response: A pilot study. Psychology Research and Behavior Management, 11, 47-54. doi: 10.2147/PRBM.S150400

Jamieson, J. P., Crum, A. J., Goyer, J. P., Marotta, M. E., \& Akinola, M. (2018). Optimizing estresse responses with reappraisal and mindset interventions: An integrated model. Anxiety, Stress \& Coping, 31(3), 245-261. doi: 10.1080/10615806.2018.1442615

JASP Team (2018). JASP (Versão 0.9) (Programa de computador). Recuperado de https://jasp-stats.org/

Kilby, C. J., \& Sherman, K. A. (2016). Delineating the relationship between estresse mindset and primary appraisals: Preliminary findings. Springer Plus, 5, 336. doi: 10.1186/s40064-016-1937
Lipp, M. E. N. (2000). Manual do inventário de sintomas de estresse para adultos de Lipp (2 ${ }^{\mathrm{a}}$ Ed.). São Paulo: Casa do Psicólogo.

Lorenzo-Seva, U., \& Ferrando, P. J. (2013).FACTOR 9.2: A comprehensive program for fitting exploratory and semiconfirmatory factor analysis and IRT models. Applied Psychological Measurement, 37(6), 497-498. doi: $10.1177 \% 2 F 0146621613487794$

Lorenzo-Seva, U., Timmerman, M.E., \& Kiers, H. A. L. (2011). The Hull method for selecting the number of common factors. Multivariate Behavioral Research, 46(2), 340-364. doi:10.1080/00273171.2011.564527

Marôco, J. (2010). Análise de equações estruturais: fundamentos teóricos, software e aplicações [Structural equation modeling: Theoretical foundations, software and applications]. Pêro Pinheiro: Report Number.

Maydeu-Olivares, A., \& Coffman, D. L. (2006). Random intercept item factor analysis. Psychological Methods, 11(4), 344-62. doi: 10.1037/1082-989X.11.4.344

Maydeu-Olivares, A., \& Steenkamp, J. B. E. M. (2018). An integrated procedure to control for common method variance in survey data using random intercept factor analysis models. Recuperado de https://www. academia.edu/36641946/An_integrated_procedure_to_control_f or_common_method_variance_in_survey_data_using_random_ intercept_factor_analysis_models

McGonigal, K. (2016). O lado bom do estresse. São Paulo: Réptil.

Muthén, L. K., \& Muthén, B. O. (2012). Mplus user's guide ( $7^{a}$ ed.). Los Angeles, CA: Muthén \& Muthén.

Park, D., Yu, A., Metz, S. E., Tsukayama, E., Crum, A., \& Duckworth, A. L. (2017). Beliefs about stress attenuate the relation among adverse life events, perceived diestresse, and self? ?control. Child Development, (1-11). doi: 10.1111/cdev.12946

Park, H., \& Hahm, S. (2019). Changes in stress mindset and EEG through e-healthcare based education. IEEE, 7, 20163-20171. doi: 10.1109/ ACCESS.2019.2895655

Paulhus, D. L. (1991). Measurement and control of response bias. In J. P. Robinson, P. R. Shaver, \& L. S. Wrightsman (Orgs.), Measures of social psychological attitudes. Measures of personality and social psychological attitudes (Vol. 1, pp. 17-59). San Diego, CA: Academic Press. doi: 10.1016/B978-0-12-590241-0.50006-X

Tabachnick, B. G., \& Fidell, L. F. (2012). Using multivariate statistics. Nova Jersey: Peason.

Valentini, F. (2017). Influência e controle da aquiescência na análise fatorial. Avaliação Psicológica, 16(2), 120-123. doi: 10.15689/ ap.2017.1602.ed

Wetzel, E., Böhnke, J. R., \& Brown, A. (2016). Response biases. In F. T. L. Leong, D. Bartram, F. M. Cheung, K. F. Geisinger, \& D. Iliescu (Orgs.), The ITC international handbook of testing and assessment (pp. 349-363). Nova lorque: Oxford University Press.

Zanon, C., Lessa, J. P. A., \& Dellazzana-Zanon, L. L. (2018). Aquiescência em autorrelatos de personalidade: uma comparação de métodos. Avaliação Psicológica, 17(4), 428-438. doi: 10.15689/ ap.2018.1704.3.03

Evandro Morais Peixoto, Doutor em Psicologia pela Pontifícia Universidade Católica de Campinas, Pós-doutor pela Universidade São Francisco - USF e Docente na mesma instituição. Endereço

para correspondência: Rua Waldemar César da Silveira, 105, Jardim Cura D'ars, Campinas, SP, CEP 13.045-510. Telefone: (19) 3779-3396. Email: peixotoem@gmail.com

Glaucia Mitsuko Ataka da Rocha, Doutora em Psicologia pela Pontifícia Universidade Católica de Campinas, Pós-doutora pelo

Estudos de Psicologia, 24(3), julho a setembro de 2019, 328-339 
Instituto de Psicologia da Universidade de São Paulo - USP, é Docente na Universidade Federal do Tocantins - UFT.

Email: gmarocha@gmail.com / glauciarocha@uft.edu.br

José de Oliveira Franco, Mestre em Psicologia pela Universidade Ibirapuera - UNIB, é Docente da Universidade Cidade de São Paulo UNICID-SP.Email: franco.jof@gmail.com e jose.franco@unicid.edu.br

José Maurício Haas Bueno, Doutor em Psicologia pela Universidade São Francisco - USF, é Docente da Universidade Federal de Pernambuco - UFPE. Email: mauricio.ufpe@gmail.com

Recebido em 18.jun.19 Revisado 08.ago.19 Aceito em 31.dez.19 